\title{
Review
}

\section{Artificial multilayers and nanomagnetic materials}

\author{
By Teruya SHINJO*1,*2,†
}

(Communicated by Shun-ichi IwASAKI, M.J.A.)

\begin{abstract}
The author has been actively engaged in research on nanomagnetic materials for about 50 years. Nanomagnetic materials are comprised of ferromagnetic systems for which the size and shape are controlled on a nanometer scale. Typical examples are ultrafine particles, ultrathin films, multilayered films and nano-patterned films. In this article, the following four areas of the author's studies are described.

(1) Mössbauer spectroscopic studies of nanomagnetic materials and interface magnetism.

(2) Preparation and characterization of metallic multilayers with artificial superstructures.

(3) Giant magnetoresistance (GMR) effect in magnetic multilayers.

(4) Novel properties of nanostructured ferromagnetic thin films (dots and wires).

A subject of particular interest in the author's research was the artificially prepared multilayers consisting of metallic elements. The motivation to initiate the multilayer investigation is described and the physical properties observed in the artificial multilayers are introduced. The author's research was initially in the field of pure physical science and gradually extended into applied science. His achievements are highly regarded not only from the fundamental point of view but also from the technological viewpoint.
\end{abstract}

Keywords: Mössbauer spectroscopy, interface magnetism, artificial multilayers with superstructures, non-coupled type GMR (giant magnetoresistance), magnetic vortex cores, domain walls in nanomagnetic wires

\section{Introduction}

The history of multilayer production is very long. In the past, X-ray spectroscopists attempted to prepare multilayers by combining heavy and light elements in order to fabricate x-ray mirrors. However, they measured only the characteristics for x-ray optics and were not concerned with the physical properties of multilayers. In general, the vacuum condition before the 1960s was not high enough to produce reliable samples. Around 1970, Esaki introduced high vacuum techniques in the field of thin film deposition and prepared semiconductor superlattices such as GaAs/AlAs. ${ }^{1}$ He showed that epitaxial multilayered structures could be fabricated with controlling the thickness in the monolayer scale.

\footnotetext{
*1 Professor Emeritus, Kyoto University.

*2 Specially-Appointed Professor, Osaka University, Osaka, Japan.

$\dagger$ Correspondence should be addressed: T. Shinjo, Institute for Chemical Research, Kyoto University, Uji, Kyoto 611-0011, Japan (e-mail: shinjoteruya@iris.eonet.ne.jp).
}

The author initiated the multilayer preparation with combining metallic elements around 1980. The necessity of multilayer samples was originated from the Mössbauer spectroscopic study on interface magnetism. The author examined the multilayer production for various combinations of metallic elements and obtained many types of multilayered structures. The artificial periodicities could be reduced to monolayer scale in some cases. Thus it was suggested that artificial multilayer preparation is a promising method to create novel materials.

\section{Mössbauer spectroscopic studies}

Mössbauer spectroscopy is a very useful tool for investigating magnetic materials because ${ }^{57} \mathrm{Fe}$ is the most convenient nucleus for Mössbauer measurements. For the study of magnetism, the most important information obtained from Mössbauer spectra is hyperfine fields, which are magnetic fields acting on the nuclei and are closely related to the local magnetic moments of $3 \mathrm{~d}$ electron spins. The Mössbauer spectra for ${ }^{57} \mathrm{Fe}$ in magnetically ordered 
materials have six-line patterns and the hyperfine field is estimated from the magnitude of the six-line splitting. In contrast, paramagnetic or non-magnetic materials show unresolved single lines, so a magnetically split spectrum is reliable evidence of the existence of magnetic order.

In 1957, R.L. Mössbauer observed for the first time that nuclei in solids can emit and absorb gamma-rays without recoil (i.e., the Mössbauer effect) and in 1961 received the Nobel Prize in Physics for his discovery of this phenomenon. The application of the Mössbauer effect to the investigation of the physical properties of solids started from around 1960, initiating the era of Mössbauer spectroscopy.

The author entered the graduate school of Science at Kyoto University in 1961 and began to use this new tool, Mössbauer spectroscopy, to the study of magnetic materials in 1962. His first target for Mössbauer spectroscopic study was an intermetallic compound, $\mathrm{Fe}_{3} \mathrm{Si}$. The spectra he obtained confirmed the existence of two hyperfine fields. This result, indicating that there are two crystallographic sites having different magnetic moments, was important to the understanding of the magnetic properties of this compound. ${ }^{2}$ Mössbauer measurement was then applied to several intermetallic compounds including $\mathrm{FeB}, \mathrm{Fe}_{2} \mathrm{~B}$ and $\mathrm{Fe}_{3} \mathrm{C}$, ${ }^{3)}$ and also to several iron oxides and hydroxides. ${ }^{4)-6)}$ This new tool proved to be very useful for characterizing the magnetic behavior of various materials containing Fe ions.

Among the author's Mössbauer studies carried out in the 1960s, a particularly important result was the measurements on ultrafine particles of magnetic compounds. In ultrafine magnetic particles with a nanometer diameter, the magnetization of each particle thermally fluctuats due to "superparamagnetism". If the rate of fluctuation is greater than the nuclear Larmor precession period, the Mössbauer spectrum does not reveal magnetic splitting, and instead is similar to a non-magnetic pattern. In 1964, the author has reported for the first time the observation of superparamagnetism by Mössbauer spectroscopy using nanoscale particles of $\alpha$-Fe $\left.\mathrm{F}_{2} \mathrm{O}_{3}{ }^{7}\right)$ and $\alpha$-FeOOH. ${ }^{8)}$ The collapse of the magnetic hyperfine structure in the Mössbauer spectra with a decrease in the particle volume was observed in the measurements. Figure 1 shows the Mössbauer absorption spectra at room temperature for $\alpha-\mathrm{FeOOH}$ ultrafine particles as a function of particle volume. Bulky samples showed a six-line pattern due to the magnetic ordering whereas the six-line pattern had

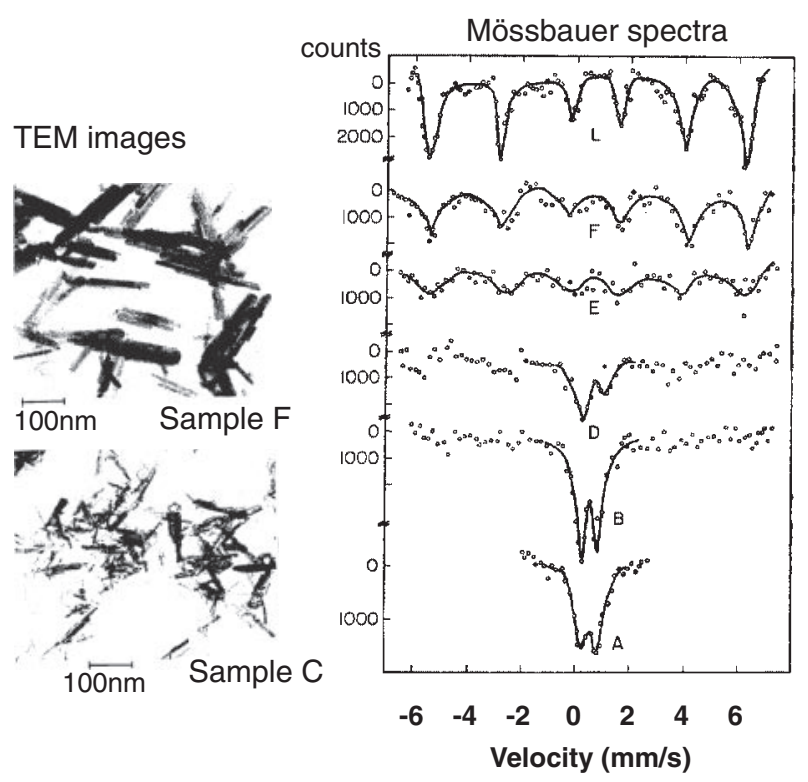

Fig. 1. Mössbauer absorption spectra at room temperature for $\alpha$ FeOOH fine particles. The particle sizes were estimated from the broadening of X-ray diffraction peaks and the average particle volumes of Samples A, B, C, D, E, F and L were estimated to be $3 \times 10^{-18}, 7 \times 10^{-18}, 1 \times 10^{-17}, 2 \times 10^{-17}, 5 \times 10^{-17}, 2 \times 10^{-16}$ and $1 \times 10^{-12} \mathrm{~cm}^{3}$, respectively. Electron micrographs for Sample $\mathrm{C}$ and $\mathrm{F}$ are also reproduced.

collapsed in the samples with smaller particles due to the occurrence of superparamagnetism. Ultrafine particles were thus the author's first research subject in the field of nanomagnetic materials.

The author's study of various magnetic materials by Mössbauer spectroscopy was done in collaboration with many research groups and demonstrated the usefulness of this new method. A particularly interesting finding was the behavior of $\mathrm{CaFeO}_{3} .{ }^{9}$ ) This compound shows a sharp single line spectrum at room temperature, which indicates that the valence state of $\mathrm{Fe}$ ions is $4+$. However, in the spectra measured at lower temperatures, two charge states with equal amounts were observed. This indicates that a charge disproportionation had taken place. From the observed Mössbauer parameters, one of the two charges is undoubtedly identified as $\mathrm{Fe}^{3+}$. Then, the other state was attributed to $\mathrm{Fe}^{5+}$. Therefore, the process of the charge disproportionation can be expressed as the following: $2 \mathrm{Fe}^{4+} \Leftrightarrow \mathrm{Fe}^{3+}+\mathrm{Fe}^{5+}$, reflecting the variation in temperature. The existence of an $\mathrm{Fe}^{5+}$ charge state is very rare in nature and no reliable example has been reported. The author's group thus obtained clear evidence that the $\mathrm{Fe}^{5+}$ state exists in $\mathrm{CaFeO}_{3}$ at low temperatures (less than $280 \mathrm{~K})$. Several compounds including $\mathrm{Fe}^{6+}$ ions were 
also studied and their magnetic properties were clarified using the results of Mössbauer measurements at various temperatures. ${ }^{10)}$ The existence of non-collinear spin structures were confirmed by the measurements in external fields for $\mathrm{CaFeO}_{3}$, as well as Kagome lattice compounds. ${ }^{5)}$

A unique feature of the author's research was the use of a stable isotope ${ }^{57} \mathrm{Fe}$ or a radioactive ${ }^{57} \mathrm{Co}$ source as a microscopic probe for investigating materials not containing $\mathrm{Fe}$ ions. A particularly successful example of this was the confirmation of antiferromagnetic order in $\mathrm{V}_{2} \mathrm{O}_{3}$ by the Mössbauer measurements using impurity ${ }^{57} \mathrm{Fe} .{ }^{11)}$ Knowledge of the existence of antiferromagnetic order is critical to the understanding of the metal-insulator transition in this compound. At that time, neutron diffraction was not yet powerful to distinguish magnetic scattering in vanadium compounds, so the information from the Mössbauer spectroscopic study of the magnetic order attracted much attention. Another strategy, the use of a radioactive isotope, ${ }^{57} \mathrm{Co}$, as a probe had been practiced in the study of impurity $\mathrm{Co}$ in the $\mathrm{Cu}$ matrix. From the Mössbauer source spectra on ${ }^{57} \mathrm{Co}$ in a $\mathrm{Cu}-2 \% \mathrm{Co}$ alloy sample, the superparamagnetic behavior was observed and the particle size distribution of precipitated Co clusters was estimated. ${ }^{12)}$

Around 1970, the author targeted "interface magnetism" for his Mössbauer spectroscopic study. In 1969 and 1970, Liebermann et al. have reported that the magnetization of an ultrathin ferromagnetic metal, $\mathrm{Fe}, \mathrm{Co}$ or $\mathrm{Ni}$, measured during the film growth is always smaller than the bulk value, and the decrease roughly corresponds to two atom layers' magnetization. Therefore they claimed that one surface layer in a ferromagnetic metal loses magnetic moment independently of the total thickness, and named it the "dead layer". 13),14) This caught the attention of both experimental physicists and theoretical physicists, and the dead layer became a hot topic. The author proposed using Mössbauer spectroscopy to verify the validity of the dead layer model. For this purpose, ${ }^{57} \mathrm{Co}$ source atoms were deposited on the surfaces of Fe and Co, by electrodeposition and the Mössbauer source spectra were measured. The observed spectra from the ferromagnetic metal surface showed magnetically split patterns for both the $\mathrm{Fe}$ and Co. ${ }^{15), 16)}$ Figure 2 is a Mössbauer source spectrum obtained using a ${ }^{57} \mathrm{Co}$ source deposited on a surface of Co metal. It shows that the surface atoms have bulk-like magnetic moments, although the hyperfine fields are fairly

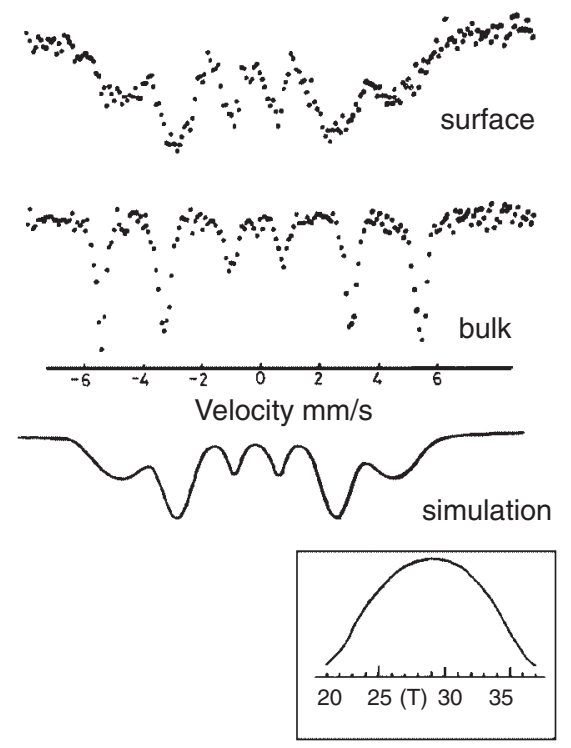

Fig. 2. Mössbauer source spectrum at $4 \mathrm{~K}$ for ${ }^{57} \mathrm{Fe}$ in the surface of Fe metal. Spectrum for the bulk Fe at $110 \mathrm{~K}$ is shown for comparison. Solid line represents calculated spectrum assuming the distribution of hyperfine field as shown in the inserted figure.

distributed and the average value is a little smaller than the bulk. This result clearly proved the nonexistence of a dead layer.

A particular advantage of using the radioactive isotope ${ }^{57} \mathrm{Co}$ is that the amount of source atoms corresponds to only $1 \%$ of the monatomic layer. Therefore if the source atoms are deposited uniformly on the substrate, the Mössbauer source spectra should furnish fruitful information about the top surface atomic layer. When ${ }^{57} \mathrm{Co}$ source is used as the sample, the Mössbauer spectra reveal the status of the daughter atom ${ }^{57} \mathrm{Fe}$, immediately after the nuclear decay. In insulating systems, the Mössbauer spectra obtained from a ${ }^{57}$ Co source sometimes show the metastable electronic state of Fe, caused by the nuclear decay. However, for metallic materials, the electronic processes are very fast, so the observed spectra should reveal the ground state electronic structure of the daughter Fe atoms.

In the initial stage of the author's investigation of interface magnetism, electrodeposition was used to prepare the source samples, because it is technically easier than a vacuum deposition. Since Liebermann et al. also used electrodeposition, the author's results were acceptable proof that the dead layer model was invalid. However, the prepared samples were frozen immediately after preparation to protect their surface from chemical reaction and therefore the measurement temperature had to be much lower 
than room temperature. The obtained Mössbauer spectra confirmed that the sample surface had retained a metallic state, but the actual surface structure could not be well characterized. A better method for preparing samples with good quality surfaces is vacuum deposition but the vacuum deposition of radioactive isotope, ${ }^{57} \mathrm{Co}$, is too challenging, so further investigation of interface magnetism was carried out by using absorber samples prepared by vacuum deposition. Namely thin film samples including the stable isotope, ${ }^{57} \mathrm{Fe}$ were prepared in ultrahigh vacuum (UHV) atmosphere and used as absorber samples for Mössbauer measurements.

\section{Interface magnetism and multilayers}

The Mössbauer effect can occur in a nucleus when the energy difference between its ground and excited states is sufficiently small. Since ${ }^{57} \mathrm{Fe}$ is fortunately the most suitable nuclear species for observing the Mössbauer effect, the ${ }^{57} \mathrm{Fe}$ Mössbauer spectroscopy has been widely used for studying magnetic materials. ${ }^{57} \mathrm{Fe}$ is one of the stable isotopes included in natural $\mathrm{Fe}$ but the natural abundance is only about $2 \%$. Natural Fe is comprised mostly of ${ }^{56} \mathrm{Fe}$, which is unrelated to the Mössbauer effect. Therefore, to obtain Mössbauer absorption spectra from a sample containing natural Fe, the Fe concentration must be significantly large. If a sample is able to be enriched in the isotope ${ }^{57} \mathrm{Fe}$, the necessary concentration of $\mathrm{Fe}$ in the sample can be greatly reduced. A designed sample for a specific research purpose can be synthesized by combining pure ${ }^{57} \mathrm{Fe}$ and pure ${ }^{56} \mathrm{Fe}$ isotopes. As will be mentioned shortly, depth-selectively enriched multilayers are typical examples of artificially designed samples. The potential of the Mössbauer spectroscopy as a tool for materials research is remarkably enhanced if ${ }^{57} \mathrm{Fe}$ isotope can be utilized. Isotopes enriched in ${ }^{57} \mathrm{Fe}$ and also ${ }^{56} \mathrm{Fe}$, up to nearly $100 \%$, can be purchased commercially, but they are very expensive.

The procedure for preparing interface-selectively enriched samples is as follows. In UHV atmosphere, a pure ${ }^{56} \mathrm{Fe}$ layer with sufficient thickness is deposited on an appropriate substrate. A Mössbauer probing layer of ${ }^{57} \mathrm{Fe}$, a few atomic layers thick, is then deposited on the ${ }^{56} \mathrm{Fe}$ layer. Finally, the surface is covered by a non-magnetic material, such as $\mathrm{V}, \mathrm{Cu}$, $\mathrm{Ag}, \mathrm{Sb}$ or $\mathrm{MgO}$. Although the prepared sample is sufficiently thick, to exhibit bulk magnetic properties, the ${ }^{57} \mathrm{Fe}$ probes are located only at the interface region of the $\mathrm{Fe}$ film. Therefore the Mössbauer

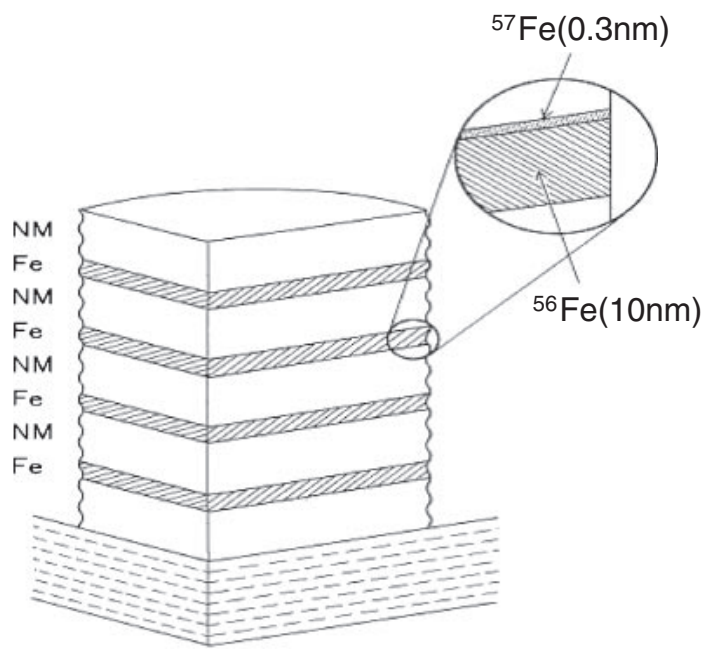

\section{Fe: Fe metal layer \\ NM: Non-magnetic layer}

Fig. 3. Structure of interface-selectively enriched sample for ${ }^{57} \mathrm{Fe}$ Mössbauer absorption spectroscopy.

spectra for such interface-selectively enriched samples should reveal the properties of the Fe interface in contact with a non-magnetic material. The structure of the interface-selectively enriched sample for the Mössbauer absorption measurements is illustrated in Fig. 3. The layer thickness is controlled by operating the shutters (see Fig. 5). In order to control the thickness in units of $0.1 \mathrm{~nm}$, the depositing rate has to be very slow. Avoidance of contamination during deposition requires the use of UHV atmosphere. The author's laboratory was the first one in Japan to use UHV deposition equipment for preparing magnetic thin films.

There are two geometries for Mössbauer measurement, transmission mode and scattering mode. Although scattering mode has a merit that the required ${ }^{57} \mathrm{Fe}$ amount in a sample can be less than the transmission mode, measurements with varying temperature and/or applying an external magnetic field are very difficult. For the study of magnetic materials, therefore, the transmission mode is better although the sample must have sufficient ${ }^{57} \mathrm{Fe}$ isotope, i.e., more than one monatomic layer. To meet this technical requirement, the author's group prepared multilayers including many ${ }^{57} \mathrm{Fe}$ layers. Namely the depositions of the [thick ${ }^{56} \mathrm{Fe} /$ ultrathin ${ }^{57} \mathrm{Fe} / \mathrm{non}-\mathrm{magnetic}$ layer] unit were repeated for many times. The use of this sample structure makes the method of the author's group for studying interface magnetism unique in the world. 


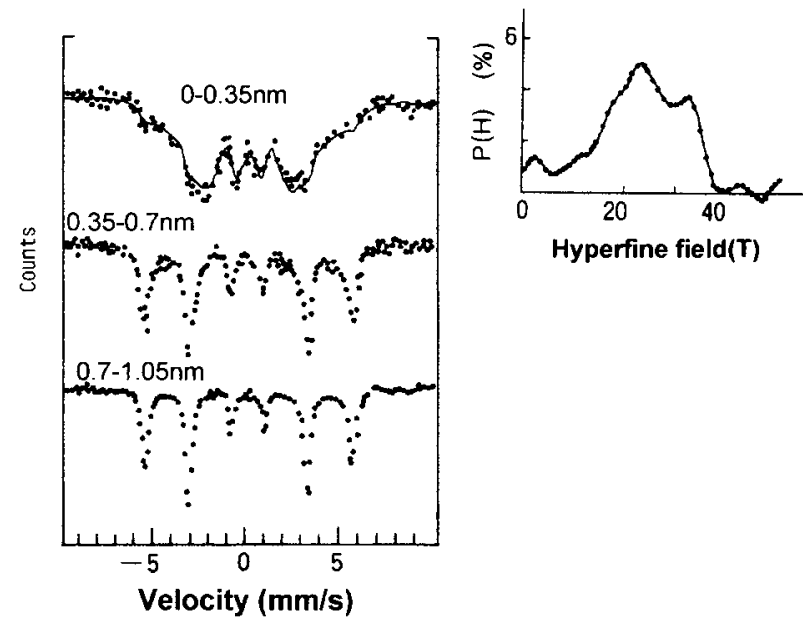

Fig. 4. Mössbauer absorption spectra at $4 \mathrm{~K}$ for samples used to study depth range from the interface of $\mathrm{Fe} / \mathrm{V}, 0-0.35 \mathrm{~nm}, 0.35-$ $0.7 \mathrm{~nm}$ and $0.7-1.05 \mathrm{~nm}$, respectively. The solid line to fit the spectrum for $0-0.35 \mathrm{~nm}$ region was drawn with assuming the distribution of hyperfined field as shown in the inserted figure.

Using interface-selectively enriched multilayer samples, the author carried out a systematic investigation of interface magnetism in $\mathrm{Fe}$ films and reached the general conclusion that the Fe metal interface is always ferromagnetic, as long as the crystallographic structure remains bcc. A minor reduction or enhancement of the local magnetic moment at the interface may be possible, but the local magnetic moment does not completely disappear. Figure 4 shows Mössbauer absorption spectra an Fe interface in contact with a non-magnetic metal, vanadium. ${ }^{17)}$ The three spectra represent the topinterface region, the $0.35-0.7 \mathrm{~nm}$ depth region, and the $0.7-1.05 \mathrm{~nm}$ depth region, respectively. The spectrum for the top interface is fairly broad and the average hyperfine field is smaller than the bulk Fe value. Nevertheless the spectrum is undoubtedly ferromagnetic and there is no dead layer. Covalent bonding with neighboring $\mathrm{V}$ atoms may reduce the Fe local magnetic moment at the interface. The spectrum for the $0.35-0.7 \mathrm{~nm}$ depth region shows a rather sharp six-line pattern and no significant deviation from that for the bulk state. The spectrum for $0.7-1.05 \mathrm{~nm}$ region is actually the same as that for the bulk state. This means that the modification of local magnetization due to the interface effect is confined to a few atomic layers.

It was also found by the author that if the surface of Fe metal is contaminated by air, the interface layer loses the magnetization at room temperature. ${ }^{18)}$ Therefore, in the case of real techni- cal magnetic materials, the magnetization of top interface layer is supposed to be significantly influenced by the condition of interface. Since the size of magnetic elements in modern devices is very small, the role of interface magnetization is crucial. As will be described later, recent studies on TMR (tunneling magnetoresistance) and spintronics revealed that the spin polarization of emitted electrons from a ferromagnetic electrode (i.e., spin current) is an important issue and it is closely related to the interface magnetization. However, nowadays, the studies on interface magnetism are not active, except those for interface anisotropy. A systematic study of magnetization in various kinds of interfaces, by means of Mössbauer spectroscopy, with combining other advanced techniques, has to be organized.

If the multilayer stacking is repeated more than 20 times, the establishment of a periodic structure in the artificial multilayer is confirmed by using X-ray or neutron diffraction techniques. Although it is difficult to microscopically examine each interface in the multilayered structure, the quality of the artificial multilayer is confirmed by the observed periodicity. The author's group prepared multilayers from various combinations of $\mathrm{Fe}$ and non-magnetic materials and found that the periodic structures can be fabricated by using a fairly wide variety of combinations. The chemical reaction and/or interdiffusion at each interface are suppressed during the multilayer film growth by depositing each layer on a cold substrate. The author's group found that periodic structures can be prepared for many combinations, independently of the phase diagram, as long as the artificial wavelength is longer than around $5 \mathrm{~nm}$. In some cases, the wavelength can be reduced to be about $2 \mathrm{~nm}$ and monatomic layers are realized as a component of multilayered structure. It was thus found that multilayers with artificial superstructures can be synthesized from various combinations of metallic elements. These materials are regarded as new materials that do not exist in nature. Besides the author's group, at that time in the world, there were several groups who were actively researching the fabrication of metallic multilayers with artificial superstructures. The main target for most of them was the creation of new superconducting materials. The author's group prepared and investigated several superconducting multilayer systems and published reports discussing the dimensionality of superconductors. ${ }^{19)-21)}$ Artificial multilayers have served as model systems for the basic study of superconductivity. However, none of 


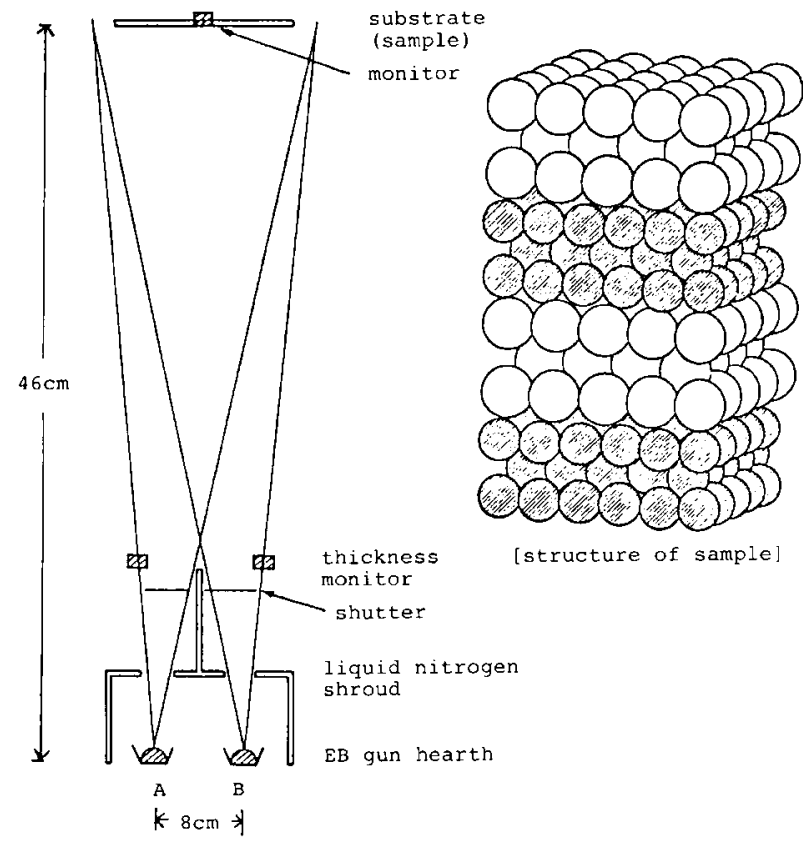

Fig. 5. Sketch of deposition system used for multilayer fabrication. Insert illustrates multilayer consisting of two metals with thickness of 3 atomic layers.

the groups had any significant success in creating novel superconducting materials.

The initial aim of the author's group was to investigate interface magnetism using multilayer structures. Around 1980, the aim switched to fabricating multilayer to create unknown materials that might have novel magnetic properties. Figure 5 shows a rough sketch of a UHV deposition system for multilayer sample preparation. A typical example of an artificially prepared new material is a multilayer structure consisting of $\mathrm{Fe}$ and $\mathrm{Mg}$ layers. ${ }^{22,23)}$ Although $\mathrm{Fe}$ and $\mathrm{Mg}$ are rather popular metal elements, they are insoluble with each other even in the liquid state. According to their binary phase diagram, they have almost no solubility with each other, so an alloy or intermetallic compound cannot be formed from $\mathrm{Fe}$ and $\mathrm{Mg}$. The author's group has synthesized $\mathrm{Fe} / \mathrm{Mg}$ multilayers by depositing $\mathrm{Fe}$ and $\mathrm{Mg}$ alternately in an UHV atmosphere on a cold substrate (cooled by liquid nitrogen). X-ray diffraction and cross-sectional TEM revealed the existence of an artificial periodic structure as shown in Fig. 6 . Visual inspection of a sample showed an artificial superstructure consisting of $\mathrm{Fe}(0.4 \mathrm{~nm})$ and $\mathrm{Mg}$ $(1.6 \mathrm{~nm})$ layers had been uniformly constructed. Structural characterization of this sample indicated that there was no epitaxial relationship in the

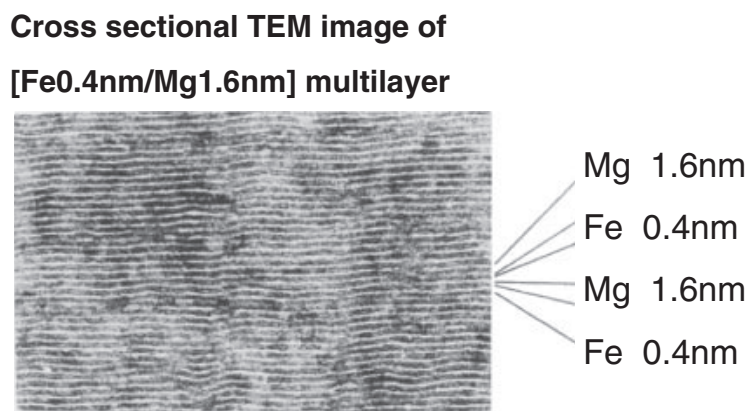

Small angle $\mathrm{X}$-ray diffraction of

[Fe1.5nm/Mg3nm] multilayer

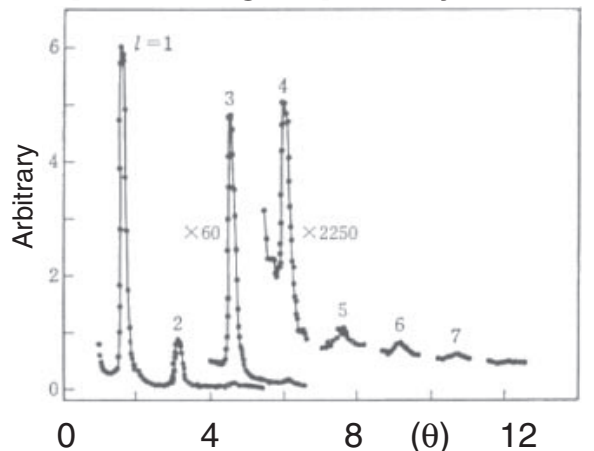

Fig. 6. Transmission electron microscopy (TEM) image for crosssection of $[\mathrm{Fe}(0.4 \mathrm{~nm}) / \mathrm{Mg}(1.6 \mathrm{~nm})]_{200}$ multilayer and smallangle x-ray diffraction (XRD) pattern for $\mathrm{Fe}(1.5 \mathrm{~nm}) / \mathrm{Mg}$ $(3 \mathrm{~nm})$ multilayer.

multilayer structure, since the atomic radii of $\mathrm{Fe}$ and $\mathrm{Mg}$ are considerably different and their intrinsic crystal structures are also different. Therefore, the microscopic structure of the $\mathrm{Fe} / \mathrm{Mg}$ multilayer structure is regarded to consist of amorphous layers. Information on the $\mathrm{Fe} / \mathrm{Mg}$ multilayer structure was also obtained from Mössbauer spectroscopic investigation as described below.

Figure 7 shows the Mössbauer absorption spectra for $\mathrm{Fe} / \mathrm{Mg}$ multilayers with various $\mathrm{Fe}$ layer thicknesses. The nominal thicknesses of individual layers are listed in the figure. The $\mathrm{Fe} / \mathrm{Mg}$ unit was deposited 30-100 times. The Mg layer thickness was $1.5-3 \mathrm{~nm}$ for all the multilayers, which is sufficient to separate individual Fe layers. Therefore from the variation of the spectra in Fig. 6, we can see that the magnetic properties are a function of the Fe layer thickness. When the thickness was $1.5 \mathrm{~nm}$, the spectra at $4.2 \mathrm{~K}$ and $300 \mathrm{~K}$ had a magnetically split sharp six-line pattern, suggesting that the Curie temperatures of the Fe layers are much high than $300 \mathrm{~K}$ and that the observed hyperfine field is equal to that of pure bcc-Fe. The profiles of spectra for Fe 


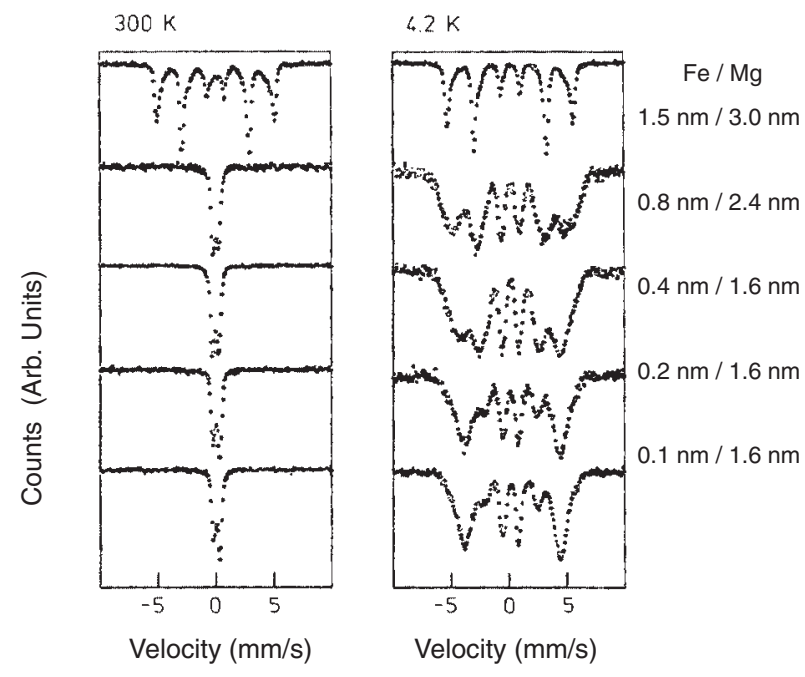

Fig. 7. Mössbauer absorption spectra at $300 \mathrm{~K}$ and $4 \mathrm{~K}$ for $\mathrm{Fe} /$ $\mathrm{Mg}$ multilayers with various Fe layer thicknesses.

layers thinner than $0.8 \mathrm{~nm}$ are completely different. At $300 \mathrm{~K}$, the spectra had no magnetically split structure and instead showed only a doublet with a small separation due to a quadrupole effect. This result indicates that the Curie temperatures of these ultrathin Fe layers are lower than $300 \mathrm{~K}$. At $4.2 \mathrm{~K}$, all spectra show magnetically split spectra but each line is very broad. This broad six-line pattern is characteristic of ferromagnetic amorphous Fe alloys. The broadening is attributed to variations in the nearest neighbor configurations.

The amorphous phase of Fe alloys in bulk form can be obtained by mixing some non-magnetic metalloid elements (such as B, C, N etc.). However, the amorphous form of pure $\mathrm{Fe}$ is known to be unstable. Extrapolation shows that the Curie temperature of amorphous pure $\mathrm{Fe}$ is remarkably low. Magnetization measurements with a SQUID magnetometer showed that the Curie temperatures of the present samples varied from about $110 \mathrm{~K}$ for the $0.8 \mathrm{~nm}$ Fe layer to about $35 \mathrm{~K}$ for the $0.1 \mathrm{~nm}$ one. Particularly interesting is the finding that the Mössbauer spectrum for the $0.1 \mathrm{~nm}$ Fe layer revealed that it was entirely ferromagnetic at $4.2 \mathrm{~K}$ and that a non-magnetic fraction was not visible. The nominal thickness of $0.1 \mathrm{~nm}$ corresponds to be less than one perfect atomic layer and thus cannot cover the entire $\mathrm{Mg}$ surface. The finding that a non-magnetic fraction was not visible indicates that the Fe atoms formed fractional monolayers and that intermixing of Fe into the $\mathrm{Mg}$ layers was negligible. Another interesting finding was that the intensity ratio of the six-line
Mössbauer pattern in the spectrum for the $1.5 \mathrm{~nm}$ Fe layer was almost ideally $3: 4: 1: 1: 4: 3$, which is expected for the case that the magnetization is oriented in the film plane. When the thickness was $0.8 \mathrm{~nm}$, the spectrum showed a structure change from bcc to amorphous but the intensity ratio was still close to $3: 4: 1: 1: 4: 3$. In contrast, as the Fe layer thickness was decreased, the intensity ratio changed gradually from $3: 4: 1: 1: 4: 3$ to $3: 0: 1: 1: 0: 3$, which is expected for the case in which the magnetization is oriented perpendicularly to the film plane. The magnetization in a thin film is typically oriented in the plane because of the shape anisotropy. On the other hand, another kind of anisotropy can exist in a surface (and interface) atom layer, "surface anisotropy," in which magnetization is oriented perpendicular to the film plane. In bulky magnetic materials, the effect of such anisotropy is negligible, because the volume fraction of the interface parts is very small. The interface anisotropy is dominant only when the magnetic layer is extremely thin, and the whole magnetization may be oriented perpendicularly. Such a situation occurs for $\mathrm{Fe} / \mathrm{Mg}$ multilayer structures if the Fe layer thickness is one or two monatomic layers.

The existence of surface (or interface) anisotropy was first demonstrated by Gradmann et al. ${ }^{24)}$ They observed that the easy direction of magnetization in FeNi ultrathin film is perpendicular to the film plane and attributed the origin to the surface anisotropy. The present author was the second to observe perpendicularly magnetized film apparently due to interface anisotropy and the first to report this finding for multilayer samples. Multilayer structures with perpendicular magnetization, which is stable even at room temperature, were realized by Philips group using samples made of $\mathrm{Co} / \mathrm{Pd}$ multilayers. ${ }^{25}$ ) Since then, multilayer structures with perpendicular magnetization have attracted much attention for potential application to magnetic recording media. Particularly promising candidates for magnetic recording media are $\mathrm{Co} / \mathrm{Pt}$ and $\mathrm{Fe} / \mathrm{Pt}$ multilayer structures.

Although the spectra shown in Fig. 7 were obtained for different samples with different Fe layer thicknesses, they can be interpreted as a sequence of spectra obtained during the growth of an Fe layer. Initially, $\mathrm{Fe}$ atoms deposited on an $\mathrm{Mg}$ substrate form fractional monolayers until there are sufficient Fe atoms to cover the whole surface. The crystal structure is regarded as amorphous but there is a stable ferromagnetic order in the Fe layer, which has 
an easy direction perpendicular to the film plane and the Curie temperature is fairly low. As the thickness of the deposited $\mathrm{Fe}$ layer approaches $0.8 \mathrm{~nm}$, the magnetization direction gradually turns toward the film plane. A drastic change in the structure from amorphous to crystalline bcc occurs between 0.8 and $1.5 \mathrm{~nm}$. The Curie temperature jumps up a much higher than room temperature in association with the crystallographic transformation. The spectra for $1.5 \mathrm{~nm}$ Fe layers revealed that they were entirely ferromagnetic and the observed hyperfine field value was the same as the pure bulk Fe value. The sharp line width indicates that the bcc Fe layer did not include $\mathrm{Mg}$ impurities. If the sample included a significant amount of $\mathrm{Mg}$ impurities, the amorphous structure would be stable and the transformation to a bcc structure would not take place. This means that the amount of $\mathrm{Mg}$ dissolved in the ultrathin amorphous Fe layer was negligible. It was therefore concluded that the structure of an ultrathin Fe layer deposited on an Mg substrate is amorphous, as long as the thickness remains below a critical value, which lies between 0.8 and $1.5 \mathrm{~nm}$. If the thickness exceeds the critical value, a sudden transformation to bcc takes place. ${ }^{26)}$ Thus, it has been demonstrated that $\mathrm{Fe} / \mathrm{Mg}$ multilayers are well suited for investigating the magnetic properties of "pure" amorphous Fe metal, although the layer thickness is limited to less than about $1 \mathrm{~nm}$.

\section{Giant magnetoresistance effect and magnetic multilayers}

During the 1980s, magnetic multilayers with artificial superstructures became the main theme of the author's research group. Moreover, in the area of magnetism research, multilayers were regarded as one of the most interesting subjects. A plenary talk presented by the author was a highlight of the International Conference on Magnetism (ICM) held at San Francisco in 1985 and magnetic multilayers gained world-wide attention. Novel physical properties were expected to be discovered in such artificially synthesized materials. Several other groups as well were preparing and investigating multilayers and many new laboratories designed for their study were opening up. The investigations focused not only on the magnetic behaviors but also on their superconducting properties. A feature of the author's work was that multilayer preparation was originally from their study of interface magnetism.

A. Fert (in Orsay) was interested in magnetic multilayers and visited the author's laboratory in
1987 to see the equipment used for multilayer synthesis and to discuss potentialities of such novel materials. After his visit, he decided to begin the experiments on multilayers and achieved great success in 1988 by using $\mathrm{Fe}$ and $\mathrm{Cr}$ as multilayer constituents. Independently, P. Grünberg and his colleagues (in Julich) were investigating the magnetic properties of the $\mathrm{Fe} / \mathrm{Cr} / \mathrm{Fe}$ sandwich systems. ${ }^{27)}$ They measured the magnetic behaviors of the system by changing the thickness of the Cr spacer layers and found a rather strong antiferromagnetic exchange interaction between two Fe layers, separated by $\mathrm{Cr}$ layers, when the Cr layer thickness was around $1 \mathrm{~nm}$. Namely the Fe layer magnetizations were spontaneously oriented antiparallel to each other and aligned parallel if a large external field was applied. They measured the magnetoresistance of $\mathrm{Fe} / \mathrm{Cr} / \mathrm{Fe}$ sandwich films and found that the resistance when the two magnetizations were antiparallel was larger than when they were parallel. This result clearly indicates that the conductance is affected by the magnetic spin structure, which is the physical principle of the GMR effect. However the observed MR ratio in the three-layer system, (about 1.5\%), was not large enough to draw a significant impact. ${ }^{28)}$

Really 'giant' magnetoresistance was first observed by the Fert's group for Fe/Cr multilayers in $1988 .^{29)}$ They intended to visualize the role of interlayer coupling using samples with a multilayered structure. They have prepared epitaxial Fe/Cr multilayers with various $\mathrm{Cr}$ layer thicknesses and the measured magnetic properties including the magnetoresistance. As reproduced in Fig. 8, surprising results were obtained for resistance measurements in external fields. For the $[\mathrm{Fe}(3 \mathrm{~nm}) / \mathrm{Cr}$ $(0.9 \mathrm{~nm})]_{\mathrm{x} 60}$ sample for instance, the resistance at $4 \mathrm{~K}$ decreased to almost a half at the saturation field. The MR ratio was nearly $20 \%$ even at room temperature, which is a strikingly large value in comparison with the conventional MR changes. It has been known that ferromagnetic alloys exhibit the anisotropic magnetoresistance (AMR) but the MR ratio is not more than a few percent at room temperature. The results of Fert's GMR experiment confirmed the existence of a strong antiferromagnetic interlayer coupling, between Fe layers separated by a Cr spacer layer.

The mechanism of the GMR was phenomenologically explained rather soon after the discovery by considering the spin-dependent scattering of conduction electrons. The scattering probability for conduction electrons at the interface of the ferromag- 


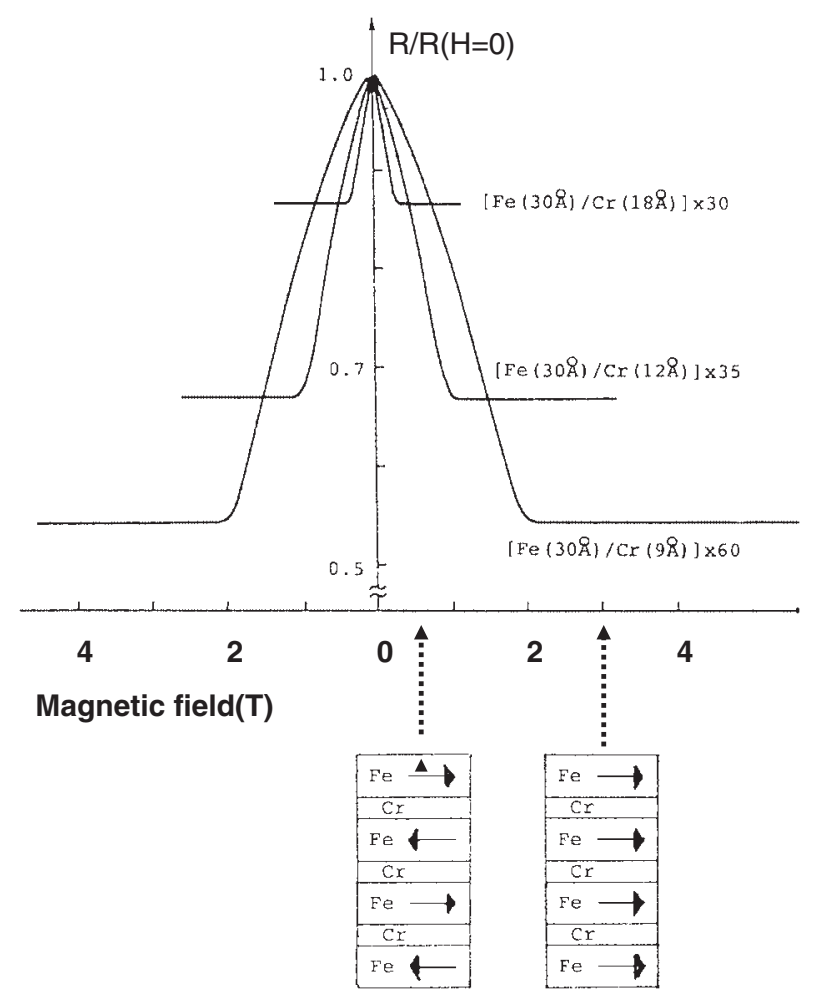

Fig. 8. Giant magnetoresistance (GMR) at $4.2 \mathrm{~K}$ of $\mathrm{Fe} / \mathrm{Cr}$ multilayers. ${ }^{29)}$

netic layer should depend on the spin direction, up or down. For instance an up-spin electron can penetrate without scattering from a Cr layer into an Fe layer having up-spin magnetization, while a down-spin electron is scattered. If the $\mathrm{Fe}$ layers have an antiparallel magnetic structure, both up- and downspin electrons soon meet an Fe layer having a magnetization in the opposite direction (within two Fe layers' distance), so the probability of scattering is high for both types of electrons. On the other hand, if all the Fe layers have parallel magnetizations, down-spin electrons are scattered at every Fe layer whereas up-spin electrons can move across a long distance without scattering. In other words, the mean-free-path of up-spin electrons can be considerably long, while that of down-spin electrons must be very short. Total conductance of the system is the sum of conductances due to up-spin and downspin electrons. Because of the long mean free path of up-spin electrons, the total resistance is much smaller in the parallel magnetization state than in the antiparallel one.

The discovery of the GMR effect raised two key issues; interlayer coupling and spin-dependent scattering. In addition for the $\mathrm{Fe} / \mathrm{Cr} / \mathrm{Fe}$ sandwich system, the existence of antiferromagnetic interlayer coupling was reported for $\mathrm{Co} / \mathrm{Cu}$ multilayers and many other systems. If the interlayer coupling is antiferromagnetic, the GMR effect is almost always observed. That is, the resistance in the antiferromagnetic state is larger than that in the ferromagnetic state. In a study of $\mathrm{Co} / \mathrm{Cu}$ multilayers with various $\mathrm{Cu}$ layer thicknesses, an amazing result was obtained: the interlayer coupling strength across the $\mathrm{Cu}$ layer oscillated with the variation in the $\mathrm{Cu}$ layer thickness. ${ }^{30}$ ) Since the MR effect is caused by the antiferromagnetic coupling, the observed MR ratio showed a periodic variation as a function of the $\mathrm{Cu}$ layer thickness. In other words, MR measurements can be used to verify that the sign of interlayer coupling is negative. Parkin et al. prepared multilayers combining $\mathrm{Co}$ and various non-magnetic metals and found that the oscillation of interlayer coupling occurs rather generally with a wavelength of $1-1.5 \mathrm{~nm}^{31)}$

The oscillatory features of simple normal metals were accounted for by considering the band structures and quantum well states, and accordingly our understanding of the electronic structures of simple metal films has been remarkably advanced.

The GMR effect is caused by the change in the magnetic structure between antiparallel and parallel alignments. In the case of $\mathrm{Fe} / \mathrm{Cr}$ and also $\mathrm{Co} / \mathrm{Cu}$ multilayers, the antiparallel configuration that originates from the antiferromagnetic interlayer exchange coupling is converted into a ferromagnetic configuration by an externally applied magnetic field. The magnitude of the external field necessary for this conversion depends on the strength of the interlayer coupling. Because of the strong interlayer coupling, the magnetic field required to induce the MR effect in $\mathrm{Fe} / \mathrm{Cr}$ multilayers is significantly large (about $2 \mathrm{~T}$ ). The coupling of $\mathrm{Co} / \mathrm{Cu}$ system is smaller but the saturation field is still too high for the MR effect to be exploited in technical applications such as magnetic recording sensors.

Another type of GMR was demonstrated by the author's group in 1990, by using non-coupled multilayer samples. ${ }^{32)}$ Multilayers comprising two magnetic elements were prepared by successively stacking $\mathrm{NiFe}(3 \mathrm{~nm}), \mathrm{Cu}(5 \mathrm{~nm}), \mathrm{Co}(3 \mathrm{~nm})$ and $\mathrm{Cu}(5 \mathrm{~nm})$ layers. Since the $\mathrm{Cu}$ spacer layers were not extremely thin, the interlayer coupling between the two magnetic layers, $\mathrm{NiFe}$ and $\mathrm{Co}$, was negligibly small and so these two layers behaved independently. While $\mathrm{NiFe}$ is a soft magnetic material, $\mathrm{Co}$ is a rather hard one, so the coercive forces for these layers 


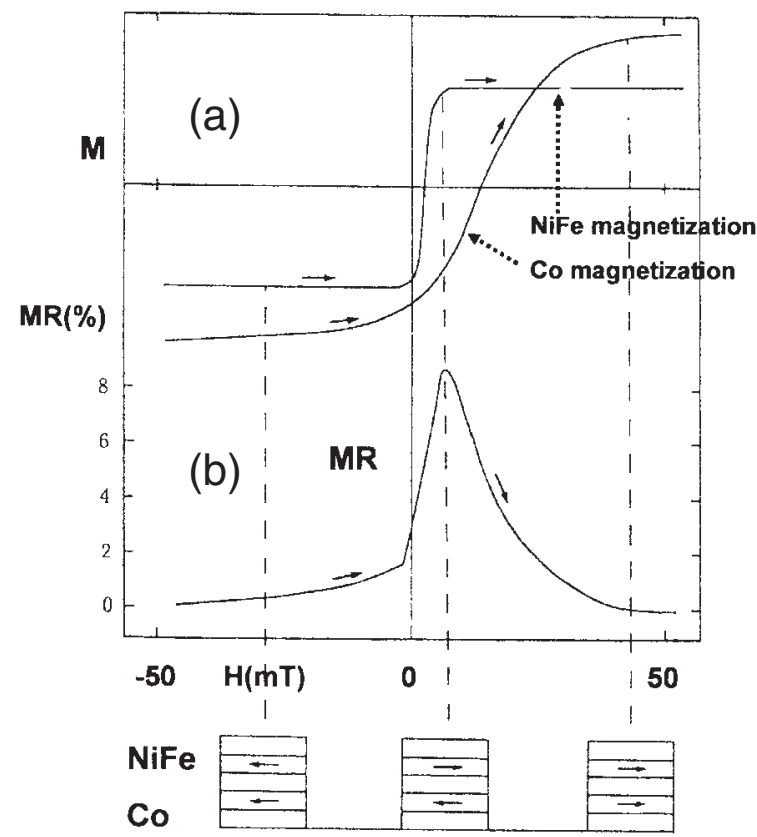

Fig. 9. Non-coupled type GMR effect of $[\mathrm{Co}(3 \mathrm{~nm}) / \mathrm{Cu}(5 \mathrm{~nm}) /$ $\mathrm{NiFe}(3 \mathrm{~nm}) / \mathrm{Cu}(5 \mathrm{~nm})]_{15}$ multilayer at $300 \mathrm{~K}$. (a) The observed magnetization curve with increasing field was decomposed into individual magnetizations of $\mathrm{Co}$ and NiFe. (b) Magnetic field dependence of resistance during the increasing field. The magnetoresistance effect occurs in a small field region.

are remarkably different. In the course of magnetic field sweeping, the magnetization in the NiFe layers flips much earlier than that in the Co layers and eventually an antiparallel alignment of magnetizations is realized. As reproduced in Fig. 9, a great enhancement in resistance (i.e., the GMR phenomenon) was observed in the field region for the induced antiparallel configuration caused by the difference in the coercive fields. This demonstration of noncoupled GMR confirms that the interlayer coupling has no direct affect on the MR properties. Namely GMR and interlayer coupling are independent issues. The establishment of an antiferromagnetic configuration in non-coupled GMR multilayer structures was confirmed by using the neutron diffraction method. The basic studies in details, on non-coupled GMR multilayers carried out by the authors group were surveyed elsewhere. ${ }^{33)}$ It is a feature of noncoupled GMR multilayers that the external field necessary to induce the MR change can be weak, in comparison to the original GMR effect caused by the interlayer coupling, which is a great advantage from the viewpoint of application.

For the non-coupled type GMR effect, the spacer layer has to be thick enough to prevent interlayer

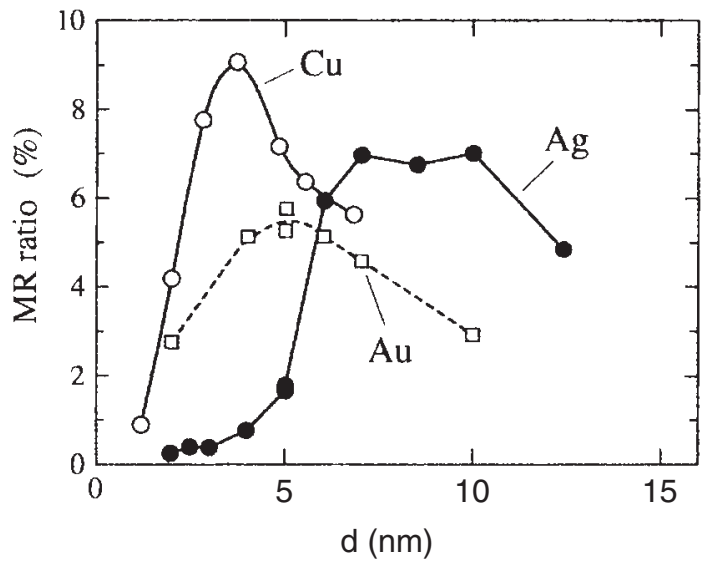

Fig. 10. Non-coupled GMR effect (MR ratio) for $\mathrm{Cu}-, \mathrm{Au}-$ and Ag-based multilayers as a function of spacer layer thickness. Magnetic layers ( $\mathrm{NiFe}$ and $\mathrm{Co}$ ) were $2.5 \mathrm{~nm}$ thick. Number of multilayer repetitions was five, for all the samples.

coupling between magnetic layers. However, if the spacer layer is too thick, the magnitude of the MR ratio is limited. The maximum $\mathrm{MR}$ ratio is obtained when the spacer layer thickness is the minimum required thickness. The MR ratio as a function of the spacer layer thickness has been examined for three normal metals, $\mathrm{Cu}, \mathrm{Ag}$ and $\mathrm{Au}$. In all the samples, the magnetic layer thicknesses ( $\mathrm{FeNi}$ and $\mathrm{Co}$ ) were $2.5 \mathrm{~nm}$ and the number of multilayer repetitions was 5. The observed MR ratio at room temperature is plotted in Fig. 10 as a function of the spacer layer thickness. ${ }^{33)}$ Although the MR ratio for Ag-based films is large in the region of thick spacer layers, the maximum value is not larger than that for $\mathrm{Cu}$-based films. This is because the critical thickness where the interlayer coupling appears is larger in Ag-based films than in $\mathrm{Cu}$-based ones. In other words, $\mathrm{Ag}$ spacer layers cannot be very thin in non-coupled GMR multilayers. This suggests that $\mathrm{Cu}$ is the best material for the spacer layer in non-coupled GMR multilayer systems. Perhaps this characteristic is not due to the intrinsic electronic structure of the normal metals but is related to the quality of the prepared interfaces. Interlayer coupling was observed in the Ag-based films, when the Ag layer thickness was thinner than $5 \mathrm{~nm}$, which suggests the existence of pin holes. If the sample quality of the Ag-based films could be improved, an MR ratio higher than that of the $\mathrm{Cu}$-based films might be obtained.

These results for non-coupled multilayers have clarified the usefulness of GMR for technical applications. Although the GMR effect is initially observed also in a three-layer structure, ferromagnetic/ 
non-magnetic/ferromagnetic, the MR ratios actually obtained for three-layer structures were not large. Multilayered structures are needed to enhance the $\mathrm{MR}$ ratio. Therefore, the experiments on multilayers were actively carried out world wide to identify the conditions under which the MR ratio is enhanced. Intensive studies on magnetic multilayers continued, for about a decade, until the appearance of the TMR effect (tunneling magnetoresistance).

The technological application of GMR effect was first realized as magnetic recording heads by using non-coupled sandwich films with two magnetic components, such as $[\mathrm{NiFe}(15 \mathrm{~nm}) / \mathrm{Cu}(2.6 \mathrm{~nm}) /$ FeNi $(15 \mathrm{~nm}) /$ FeMn $(10 \mathrm{~nm})]$, by IBM group. ${ }^{34)}$ An antiferromagnetic FeMn layer is attached to one of the NiFe layer to increase the coercive force. while the other NiFe layer behaves freely as a soft magnet. These magnetic layers are called the "pinned layer" and the "free layer", respectively. Although the $\mathrm{MR}$ ratio of the spin-valve system is not large, satisfactorily high magnetoresistance sensitivity was achieved.

Concerning enhancement of the MR ratio, the next breakthrough was obtained in the studies of tunneling magnetoresistance, TMR. ${ }^{35), 36)}$ In TMR systems, the non-magnetic metal spacer layer in a non-coupled GMR system was replaced by insulator spacer layer (i.e., tunneling barrier). In 1996, an MR ratio of about $18 \%$ at $300 \mathrm{~K}$ was reported. In 2004, using $\mathrm{MgO}$ as the material for the tunneling barrier, an MR ratio of more than $100 \%$ was obtained. ${ }^{37), 38)}$

In recent computers, the capacity of magnetic recording has been greatly advanced by adopting perpendicular magnetic recording method invented by S. Iwasaki. ${ }^{39)}$ In order to analyze information from magnetic recording with extremely high density, read-out heads are required to have enough sensitivity for very small field variation. Magnetoresistive sensors using the non-coupled GMR principle (i.e., GMR head and TMR head) fulfill this requirement and are actually being used in commercial computers.

The discovery of the GMR effect was an especially important one from both the fundamental and application viewpoints. This is evidenced by the awarding of the Nobel Prize in Physics in 2007 to A. Fert and P. Grünberg for its discovery.

On the other hand, concerning further development of magnetic multilayer studies, it is expected that the potential use of perpendicularly magnetized films (e.g., $\mathrm{Co} / \mathrm{Pt}$ multilayers) as recording media materials will be explored in the near future.

\section{Nanostructured magnetic systems}

The author's group started to use electron-beam lithography to prepare nanostructured magnetic materials (i.e., dots and wires), in the mid-1990's. They were one of the first groups to use nanofabrication techniques for sample preparation in the field of magnetism. They initially focused on using nanofabrication techniques to prepare GMR samples with a columnar structure. A strategy to obtain an enhanced GMR effect was to use CPP geometry (with current perpendicular to the film plane). Since the resistance of thin metallic films in the direction perpendicular to the film plane is extremely low, columnar samples with nanoscale dimensions are required for $\mathrm{CPP}$ measurements. However, the results of their CPP measurements obtained in the author's laboratory were not noteworthy. In contrast, their use of nanofabrication techniques to prepare nanodots and nanowires produced outstanding results regarding magnetic vortices in NiFe dots and the dynamic behavior of magnetic domain walls in $\mathrm{NiFe}$ nanowires.

(a) Magnetic vortex core. Ferromagnetic materials generally form domain structures to reduce the magnetostatic energy. In small ferromagnetic systems, however, it is not energetically preferable to form domain walls, so a single domain structure may be stabilized in a ferromagnetic thin film in a small area. Another possible spin structure for a nanoscale magnetic thin film, typically for a circular dot, is a curling spin configuration (magnetization vortex). The concept of magnetization vortex structure is illustrated in Fig. 11. In a magnetic thin film, the spins tend to lie in the sample plane due to shape anisotropy. A vortex is a favorable spin structure, since spin directions change gradually in the plane so that exchange energy is not lost but the total dipole energy is canceled out. However, the angle between adjacent spins becomes significantly large in the vicinity of the central part, as long as the spin directions are confined to the film plane. This means that the magnetizations in a small spot at the center of the vortex structure should be perpendicular to the plane. Although this hypothesis was proposed long ago, there had been no direct evidence reported for the existence of a perpendicularly magnetized vortex core. The author's group succeeded in observing perpendicularly magnetized vortex cores by using magnetic force microscopy (MFM), for the first time. ${ }^{40)}$ They prepared NiFe dots $(1 \mu \mathrm{m}$ diameter and $50 \mathrm{~nm}$ thick) and obtained MFM image as shown 

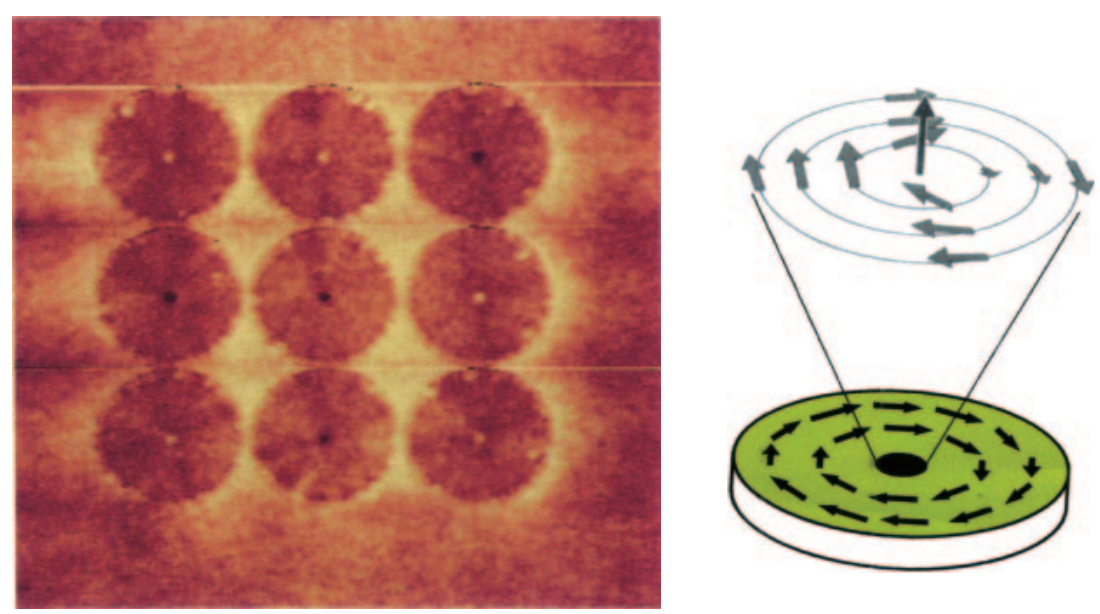

Fig. 11. Magnetic force microscopy (MFM) image of array of NiFe dots (1 $\mu \mathrm{m}$ in diameter and $50 \mathrm{~nm}$ thick). Schematic spin structure of magnetic vortex and vortex core in a dot is also illustrated.

in Fig. 11. In the MFM image, each dot does not show the usual domain pattern but their profiles are rather uniform, except for a small spot at the center of each dot. This suggests that a conventional magnetic domain pattern does not exist in each dot and that a vortex structure has been formed. The vortex cores were either white or dark with random distribution. When an external field was applied, the core color was unified, since the direction of core magnetization was oriented in the field direction. This is clear evidence that the small spots observed in the MFM image correspond to the perpendicularly magnetized vortex cores and each magnetization was randomly oriented in the initial stage.

Core magnetization switching was studied by applying an external field to arrays of $\mathrm{NiFe}$ dots with a diameter of $400 \mathrm{~nm}$, and then examining the MFM images. The magnetic field necessary for complete reorientation of the core magnetizations depended on the direction of the magnetic field. For the perpendicular direction, the saturation field was about $0.4 \mathrm{~T}$. For the in-plane direction, the core starts to move and was annihilated at about $0.1 \mathrm{~T} .{ }^{41}$ ) The dynamic behavior of the core due to an electric current was recently examined. Namely, application of a high frequency ac current caused the core to show resonant behavior and to move along a circular orbit. Furthermore, it was recently observed that a nanosecond single current pulse can switch the core magnetization. This suggests that the magnetic dots with vortex magnetization are potentially applicable to magnetic recording memories. ${ }^{42)}$

(b) Domain walls in nanowire. Using nanofabrication techniques, nanowire systems of ferro- (a)

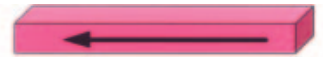

Magnetization reversal

(b) =domain wall movement

(c)
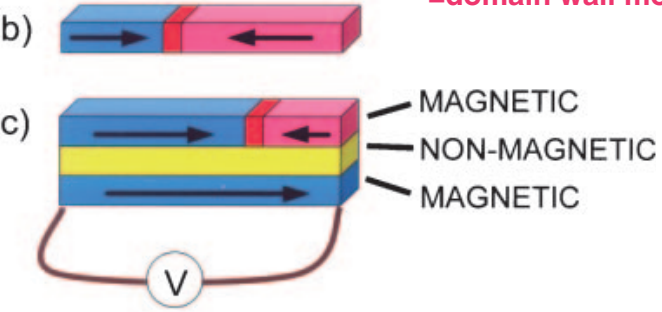

(d)

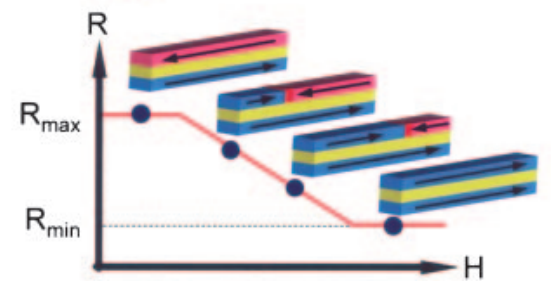

Fig. 12. Schematic illustration of the magnetization reversal process in a magnetic nanowire. Domain wall propagation was detected from resistance measurement using GMR principle.

magnetic materials, typically $\mathrm{FeNi}$ (permalloy), were prepared and the dynamic properties of their domain walls were comprehensively investigated. As illustrated in Fig. 12(a), a nanoscale wire tends to have a single domain structure and its magnetization is preferably oriented in the long axis direction, due to shape anisotropy. If an external field is applied in the opposite direction, a magnetization reversal takes place with the displacement of a domain wall and the intermediate stage during the magnetization reversal should be as shown Fig. 12(b). The dynamic behavior of a single domain wall was studied by preparing 
a sample with a trilayer structure as shown in Fig. 12(c) and using it for resistance measurement. The trilayer structure consisted of two ferromagnetic layers and one nonmagnetic spacer layer. The electric current mainly flowed in the non-magnetic conductive layer $(\mathrm{Cu}$ in this case), but the total resistance depended on the magnetic configuration of the adjacent ferromagnetic layers, i.e., parallel or antiparallel. This is the principle of GMR effect. The resistance is proportional to the relative volume of the antiferromagnetic configuration. If one of the magnetizations is stable, the position of domain wall in the other magnetic layer can be determined from the resistance as illustrated in Fig. 12(d).

The velocity of a domain wall in a nanowire, when driven by a magnetic field was estimated from the resistance measurements using a rather long (2 mm) trilayer sample. ${ }^{43)}$ The trilayer structure was $\mathrm{NiFe}(5 \mathrm{~nm}) / \mathrm{Cu}(20 \mathrm{~nm}) / \mathrm{NiFe}(40 \mathrm{~nm})$ and the wire width was $0.5 \mu \mathrm{m}$, as illustrated in Fig. 13. The result of MR measurement, the resistance as a function of the external field, is shown in Fig. 14(a). Before the field sweeping, an external field of $50 \mathrm{mT}$ was applied to align the two magnetizations in one direction and afterwards an external field was applied in the opposite direction, increasing at a rate of $2 \mathrm{mT} / \mathrm{s}$. The resistance was enhanced in the field region of 3.5 to $8.5 \mathrm{mT}$, owing to the non-coupled GMR effect. The increase at around $3.5 \mathrm{mT}$ corresponds to the reversal of the magnetization in the thinner $\mathrm{NiFe}$ layer. This change did not occur very quickly, since the external field was moderate and domain wall pinning may have occurred in the wire. In contrast, the decrease in the resistance at about 85 Oe occurred very quickly, due to the magnetization reversal in the thicker NiFe layer. The displacement of the domain wall was found to proceed very quickly. To analyze the magnetization reversal in detail and to estimate the velocity of the domain wall propagation in the thicker NiFe layer, a digital oscilloscope was used to monitor the fast resistance change at about $8.5 \mathrm{mT}$ and the result is reproduced in Fig. 14(b). It should be noted that the observed resistance change was almost linear with time, so the velocity is regarded as a constant. The propagation over the distance of $2 \mathrm{~mm}$ has taken the time of $13 \mu \mathrm{s}$. Therefore the velocity is derived to be $154 \mathrm{~m} / \mathrm{s}$. In the time interval of the velocity measurement, the change in the applied magnetic field, about $8.5 \mathrm{mT}$, was extremely small and thus regarded as a constant. In short, the dependence of domain wall velocity in a nanowire on the external field has been systematically studied.
It is natural that a domain wall can be driven by applying a magnetic field. However until recently it was not clear whether an electric current could drive a domain wall. Current-driven domain wall propagation is an interesting topic because if it is a controllable phenomenon, it may have a great importance from a technical point of view. Ono's group used MFM technique to observe domain wall displacement and succeeded in observing a domain wall being driven by an electric current (Fig. 15). ${ }^{44)}$ They identified the position of domain wall in a $\mathrm{NiFe}$ nanowire and measured its displacement as a function of the current pulse duration. Figure 16 shows the domain wall displacement against pulse duration. From these data, the velocity of the domain wall was estimated to be $3 \mathrm{~m} / \mathrm{s}$, demonstrating that a domain wall can apparently be driven by an electric current, although it is much slower than the fielddriven case. The major driving force is attributed to the spin-torque of the electric current flowing in the ferromagnetic wire.

Magnetization reversal is a key phenomenon for magnetic devices and the understanding of dynamical domain wall behavior is very important for this phenomenon. It is usual that the displacement of domain wall is driven by magnetic field and then the magnetization reversal is induced. However, current driven magnetization reversal is advantageous in terms of energy consumption and therefore it will be adopted in technical applications in future. The present result is the first observation for currentdriven domain wall displacement in nanomagnetic system. In comparison with the field-driven case, current driven magnetization reversal is advantageous also to enhance the density of magnetic memories. At the moment, the necessary current density is very high and the velocity of domain wall displacement is very slow. Nevertheless, current driven domain wall motion is an attractive research area for further development of spintronics.

\section{Remarks}

The author initially focused his research on fine particles. Using Mössbauer spectroscopy, he investigated the dynamic behavior of superparamagnetic particles. Nanoparticle samples of Fe oxide and hydroxide were prepared as precipitates in aqueous solution. The particles in solution grew in a self-organized manner, so the particle size could not be artificially controlled. Instead, it was regulated by adjusting the conditions of chemical reaction. 


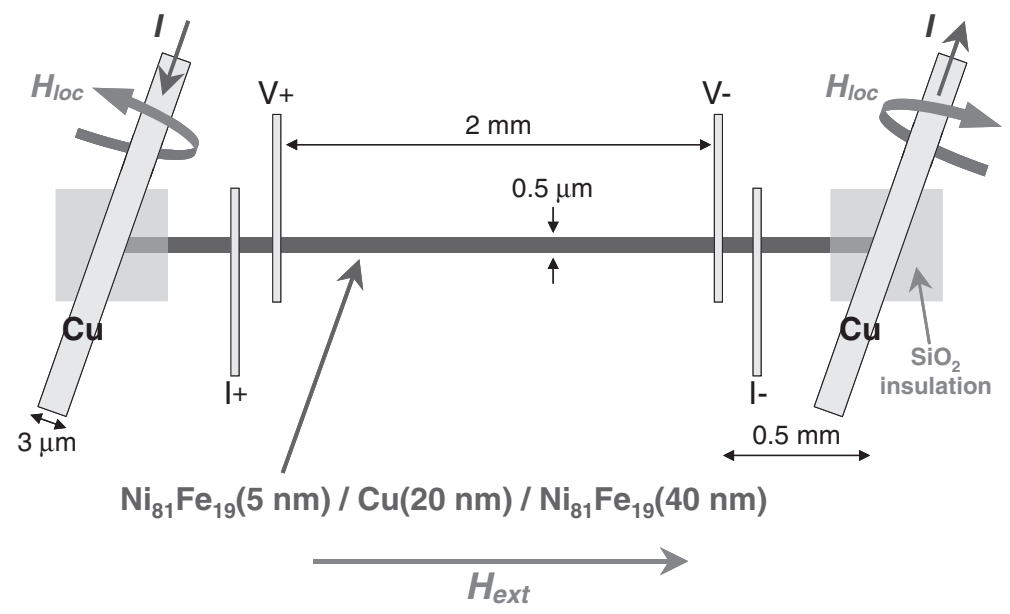

Fig. 13. Structure of trilayer magnetic wire consisting of $[\mathrm{NiFe}(5 \mathrm{~nm}) / \mathrm{Cu}(2 \mathrm{~nm}) / \mathrm{NiFe}(40 \mathrm{~nm})]$ used for measurement of domain wall propagation. Outside $\mathrm{Cu}$ wires were used to generate local magnetic field to nucleate magnetic domain wall.

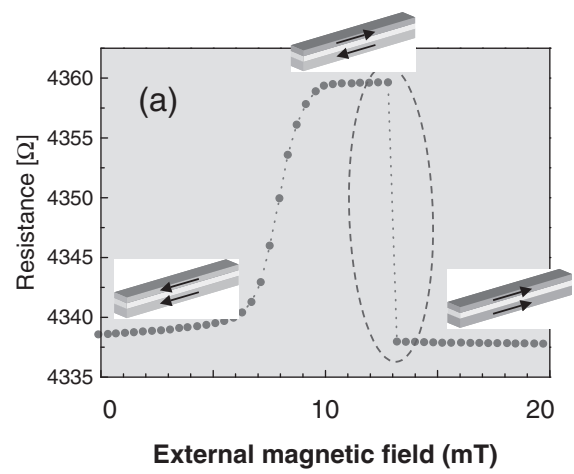

Velocity estimation: $2 \mathrm{~mm} \div 13 \mu \mathrm{s}=154 \mathrm{~m} / \mathrm{s}$
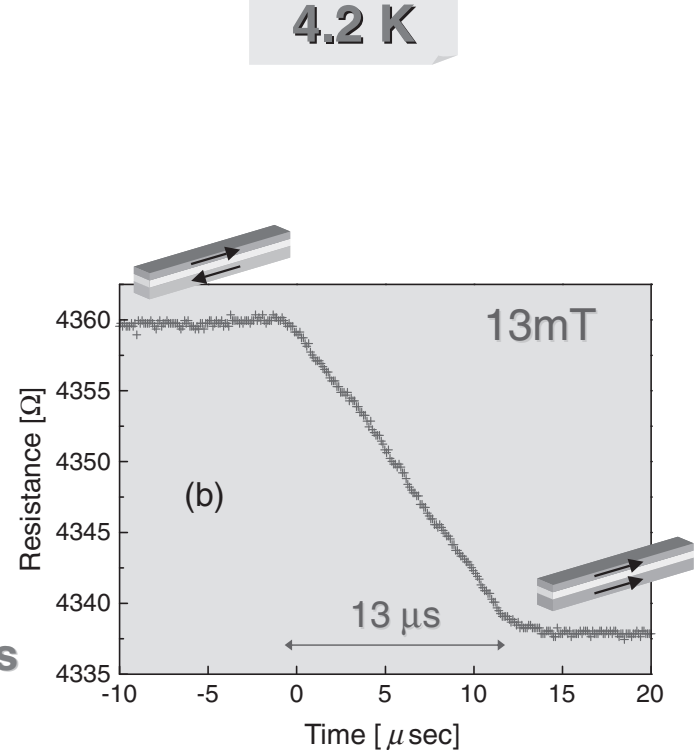

Fig. 14. (a) Resistance as a function of the external magnetic field at $4 \mathrm{~K}$ for the sample shown in Fig. 13. Field sweeping rate was $2 \mathrm{mT} / \mathrm{s}$. (b) Time variation of the resistance during the magnetization reversal of the $\mathrm{NiFe}(40 \mathrm{~nm})$ layer. Applied field was practically constant during the measurement, $13 \mathrm{mT}$.

To study ferromagnetic metal interfaces, interface-selectively enriched samples, combining two stable isotopes, ${ }^{56} \mathrm{Fe}$ and ${ }^{57} \mathrm{Fe}$, were prepared by ultrahigh vacuum (UHV) deposition. The film thickness was controllable to the atomic scale, This technique for UHV deposition was used to fabricate multilayers with artificial superstructures containing two or more elements. Such multilayers are regarded as new materials that do not exist in nature. It was therefore expected that they would exhibit novel physical properties. In fact, the giant magnetoresistance (GMR) effect was discovered using magnetic multilayers.

GMR was first observed in 1988, in $\mathrm{Fe} / \mathrm{Cr}$ multilayers by a French group. Soon thereafter, the author's group prepared non-coupled GMR multilayers, that exhibited the GMR effect in a relatively small magnetic field region, demonstrating 

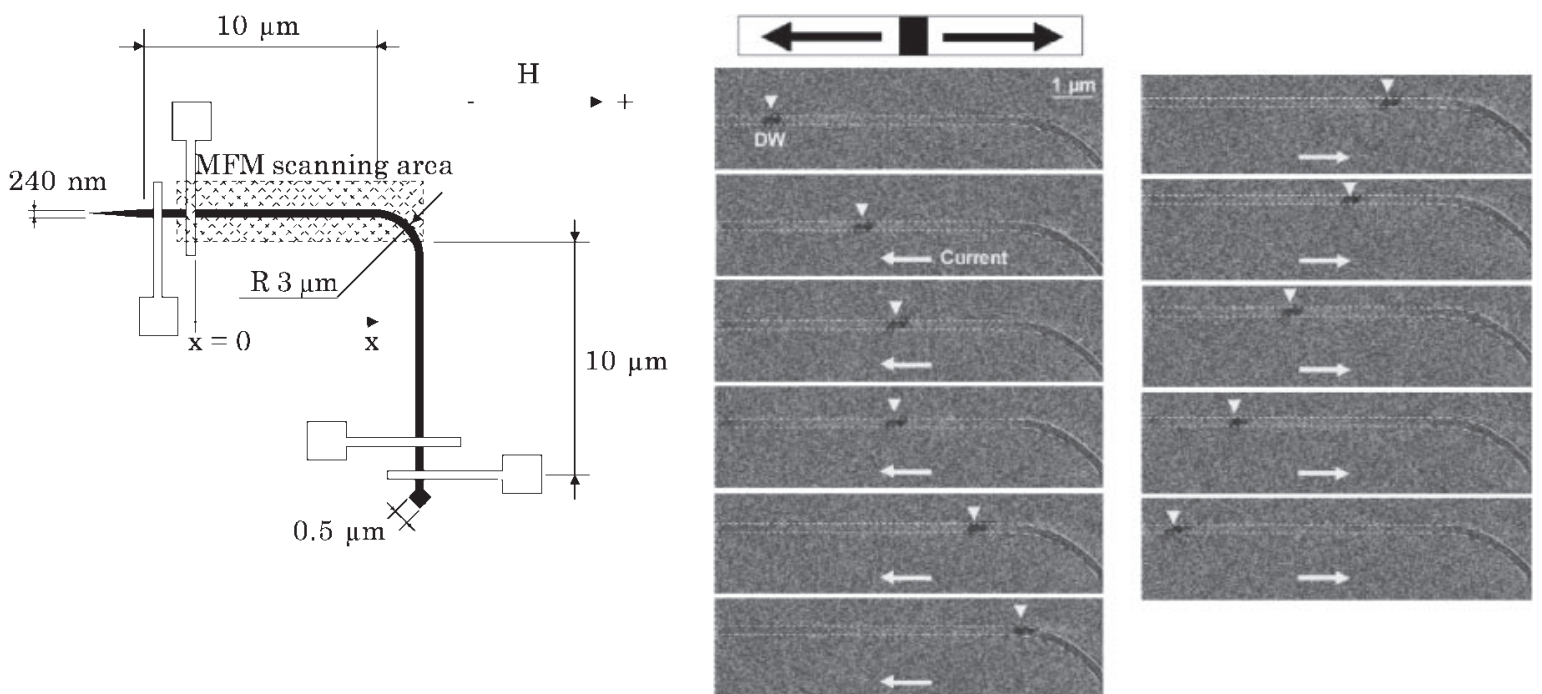

Fig. 15. MFM images captured for successive applications of one pulse current. Current density and the pulse duration were $7 \times$ $10^{11} \mathrm{~A} / \mathrm{m}^{2}$ and $0.5 \mu \mathrm{s}$, respectively. Domain wall location is marked by arrowhead.

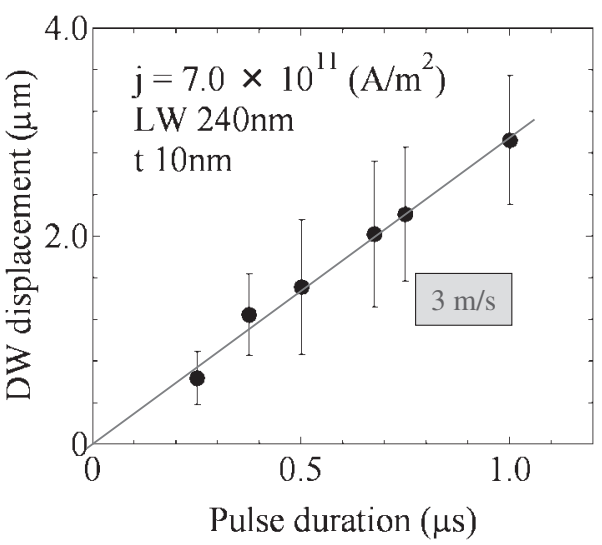

Fig. 16. Average domain wall displacement per one pulse current as a function of pulse duration.

its potential for technical application. Actually, magnetoresistance sensors, using the principle of non-coupled GMR effect, are serving as magnetic recording heads for commercial use.

Using electron-beam lithography, the authors group prepared nano-dots and wires of ferromagnetic thin films and studied the dynamic behavior of magnetic vortices in dots and of domain walls in wires. The velocity of domain wall motion, driven by electric current, in a nanomagnetic system, has been estimated for the first time.

\section{Acknowledgements}

The author retired from Kyoto University in 2002. His research activities on nanomagnetic mate- rials have been continued by his successor, Prof. T. Ono and his coworkers. Remarkable progress has been made on clarifying the dynamic properties of the magnetic vortex core, and domain wall motions. ${ }^{45)}$ Since 2005, the author has kept in touch with Prof. Y. Suzuki and Prof. M. Shiraishi at Osaka University, supporting their research on nanomagnetism and spintronics. The results of their collaboration have been presented in several publications. ${ }^{46)-48)}$

\section{References}

1) Esaki, L. and Chang, L.L. (1974) New transport phenomenon in a semiconductor superlattice. Phys. Rev. Lett. 33, 495-498.

2) Shinjo, T., Nakamura, Y. and Shikazono, N. (1963) Magnetic study of $\mathrm{Fe}_{3} \mathrm{Si}$ and $\mathrm{Fe}_{5} \mathrm{Si}_{3}$ by Mössbauer effect. J. Phys. Soc. Jpn. 18, 797-801.

3) Shinjo, T., Itoh, F., Takaki, H. and Nakamura, Y. (1964) $\mathrm{Fe}^{57}$ Mössbauer Effect in $\mathrm{Fe}_{2} \mathrm{~B}, \mathrm{FeB}$ and $\mathrm{Fe}_{3}$ C. J. Phys. Soc. Jpn. 19, 1252.

4) Takada, T., Kiyama, M., Bando, Y., Nakamura, T., Shiga, M., Shinjo, T., Yamamoto, N., Endoh, Y. and Takaki, H. (1964) Mössbauer study of $\alpha-, \beta$ and $\gamma$-FeOOH. J. Phys. Soc. Jpn. 19, 1744.

5) Miyamoto, H., Shinjo, T., Kiyama, M. and Takada, T. (1968) Mössbauer effect in $\mathrm{Fe}(\mathrm{OH})_{2}$. J. Phys. Soc. Jpn. 23, 1427-1430.

6) Takano, M., Shinjo, T., Kiyama, M. and Takada, T. (1968) Magnetic properties of $\mathrm{RFe}_{3}(\mathrm{OH})_{6}\left(\mathrm{SO}_{4}\right)_{2}$. J. Phys. Soc. Jpn. 25, 902-906.

7) Nakamura, T., Shinjo, T., Endoh, Y., Yamamoto, N., Shiga, M. and Nakamura, Y. (1964) $\mathrm{Fe}^{57}$ Mössbauer effect in ultrafine particles of $\alpha-\mathrm{Fe}_{2} \mathrm{O}_{3}$. Phys. Lett. 12, 178-179.

8) Shinjo, T. (1966) Mössbauer effect in antiferromag- 
netic fine particles. J. Phys. Soc. Jpn. 21, 917-922.

9) Shinjo, T., Hosoito, N., Takada, T., Takano, M. and Takeda, Y. (1980) Mössbauer spectroscopic studies on $\mathrm{CaFeO}_{3}$. Proc. Inter. Conf. Ferrites, Kyoto, pp. 393-396.

10) Shinjo, T., Ichida, T. and Takada, T. (1969) Internal magnetic field at $\mathrm{Fe}^{57}$ in hexavalent state. J. Phys. Soc. Jpn. 26, 1547-1550.

11) Shinjo, T. and Kosuge, K. (1966) Mössbauer effect study of $\mathrm{V}_{2} \mathrm{O}_{3}$. J. Phys. Soc. Jpn. 21, 2622-2626.

12) Nasu, S., Murakami, Y., Nakamura, Y. and Shinjo, T. (1968) Mössbauer effect study in $\mathrm{Cu}-2 \% \mathrm{Co}$ alloys. Scripta Met. 2, 647-648.

13) Liebermann, L.N., Fredkin, D.R. and Shore, H.B. (1969) Two-dimensional "ferromagnetism" in iron. Phys. Rev. Lett. 22, 539-542.

14) Liebermann, L.N., Clinton, J., Edwards, D.M. and Mathon, J. (1970) Dead layers in ferromagnetic transition metals. Phys. Rev. Lett. 25, 232-235.

15) Shinjo, T., Matsuzawa, T., Takada, T., Nasu, S. and Murakami, Y. (1971) Mössbauer evidence against the existence of magnetically dead layers. Phys. Lett. A 36, 489-490.

16) Shinjo, T., Matsuzawa, T., Takada, T., Nasu, S. and Murakami, Y. (1973) Surface state of ferromagnetic cobalt metal by Mössbauer spectroscopy. J. Phys. Soc. Jpn. 35, 1032-1035.

17) Hosoito, N., Kawaguchi, K., Shinjo, T., Takada, T. and Endoh, Y. (1984) Magnetic properties of Fe-V multilayered films with artificial superstructures. J. Phys. Soc. Jpn. 53, 2659-2667.

18) Shinjo, T., Shigematsu, T., Hosoito, N., Iwasaki, T. and Takada, T. (1982) Very thin oxide layer on $\mathrm{Fe}$ surface by Mössbauer spectroscopy. Jpn. J. Appl. Phys. 21, L220-L222.

19) Kanoda, K., Mazaki, H., Yamada, T., Hosoito, N. and Shinjo, T. (1986) Dimensional crossover and commensurability effect in $\mathrm{V} / \mathrm{Ag}$ superconducting multilayers. Phys. Rev. B 33, 2052-2055.

20) Kanoda, K., Mazaki, H., Hosoito, N. and Shinjo, T. (1987) Upper critical field of V-Ag multilayered superconductors. Phys. Rev. B 35, 415-418.

21) Kanoda, K., Mazaki, H., Hosoito, N. and Shinjo, T. (1987) Magnetic-field penetration depth and material parameters of $\mathrm{V}-\mathrm{Ag}$ multilayered superconductors. Phys. Rev. B 35, 8413-8420.

22) Kawaguchi, K., Yamamoto, R., Hosoito, N., Shinjo, T. and Takada, T. (1986) Magnetic properties of Fe-Mg artificial superstructure films. J. Phys. Soc. Jpn. 55, 2375-2383.

23) Shinjo, T., Hosoito, N., Kawaguchi, K., Nakayama, N., Takada, T. and Endoh, Y. (1986) Magnetic properties of artificial metallic superlattices. J. Magn. Magn. Mater. 54-57, 737-742.

24) Gradmann, U. (1974) Magnetism in oligastomic films. J. Appl. Phys. 40, 1182-1186.

25) Carcia, P.F., Meinhaldt, A.D. and Suna, A. (1985) Perpendicular magnetic anisotropy in $\mathrm{Pd} / \mathrm{Co}$ thin films. Appl. Phys. Lett. 47, 178-181.

26) Shinjo, T. (1991) Amorphous-to-crystalline transformation of ultrathin Fe layers: ${ }^{57} \mathrm{Fe}$ Mössbauer study of artificial multilayers. Struct. Chem. 2
(73), 281-288.

27) Grünberg, P., Schreiber, R., Pang, Y., Brodsky, M.B. and Sowers, H. (1986) Layered magnetic structures; evidence for antiferromagnetic coupling of Fe layers across Cr interlayers. Phys. Rev. Lett. 57, 2442-2445.

28) Binasch, G., Grünberg, P., Saurenbach, F. and Zinn, W. (1989) Enhanced magnetoresistance in layered magnetic structures with antiferromagnetic interlayer exchange. Phys. Rev. B 39, 4828-4830.

29) Baibich, M.N., Broto, J.M., Fert, A., Nguyen Van Dau, F., Petroff, F., Eitenne, P., Creuzet, G., Friederch, A. and Chazelas, J. (1988) Giant magnetoresistance of (001)Fe/(001) $\mathrm{Cr}$ magnetic superlattices. Phys. Rev. Lett. 61, 2472-2475.

30) Mosca, D.H., Petroff, F., Fert, A., Schroeder, P.A., Pratt, W.P. Jr. and Loloee, R. (1991) Oscillatory interlayer coupling and giant magnetoresistance in $\mathrm{Co} / \mathrm{Cu}$ multilayers. J. Magn. Magn. Mater. 94, L1-L5.

31) Parkin, S.S.P. (1991) Systematic variation of the strength and oscillation period of indirect magnetic exchange coupling through the $3 \mathrm{~d}, 4 \mathrm{~d}$ and $5 \mathrm{~d}$ transition metals. Phys. Rev. Lett. 64, 2304-2306.

32) Shinjo, T. and Yamamoto, H. (1990) Large magnetoresistance of field-induced giant ferrimagnetic multilayers. J. Phys. Soc. Jpn. 59, 3061-3064.

33) A review paper on non-coupled GMR multilayers is e.g.; Shinjo, T. (2002) Experiments of giant magnetoresistance. In Spin Dependent Transport in Magnetic Nanostructures (eds. Maekawa, S. and Shinjo, T.). Taylor and Francis, New York and London, pp. 1-46.

34) Dieny, B., Speriosu, V.S., Parkin, S.S.P., Gurney, B.A., Wilhoit, D.R. and Mauri, D. (1991) Giant magnetoresistance in soft ferromagnetic multilayers. Phys. Rev. B 43, 1297-1300.

35) Miyazaki, T. and Tezuka, N. (1995) Giant magnetic tunneling effect in $\mathrm{Fe} / \mathrm{Al}_{2} \mathrm{O}_{3} / \mathrm{Fe}$ junction. J. Magn. Magn. Mater. 151, 403-410.

36) Moodera, J.S., Kinder, L.R., Wong, T.M. and Meservey, R. (1995) Large magnetoresistance at room temperature in ferromagnetic thin film junctions. Phys. Rev. Lett. 74, 3273-3277.

37) Yuasa, S., Nagahama, T., Fukushima, A., Suzuki, Y. and Ando, K. (2004) Giant room temperature magnetoresistance in single crystal magnetoresistance of $\mathrm{Fe} / \mathrm{MgO} / \mathrm{Fe}$ MTJ. Nat. Mater. 3, 868-869.

38) Parkin, S.S.P., Kaiser, C., Panchula, A., Rice, P.M., Hughes, B., Samant, M. and Yang, S.H. (2004) Giant tunneling magnetoresistance at room temperature with $\mathrm{MgO}$ tunneling barrier. Nat. Mater. 3, 862-867.

39) Iwasaki, S. (1980) Perpendicular magnetic recording. IEEE Trans. Magn. 16, 71-76.

40) Shinjo, T., Okuno, T., Hassdorf, R., Shigeto, K. and Ono, T. (2000) Magnetic vortex core observation in circular dots of permalloy. Science 289, 930932.

41) Okuno, T., Shigeto, K., Ono, T., Mibu, K. and Shinjo, T. (2002) MFM study of magnetic vortex cores in circular permalloy dots; Behavior in 
external field. J. Magn. Magn. Mater. 240, 1-6.

42) Ono, T. and Shinjo, T. (2009) Dynamics of magnetic domain walls in nanomagnetic systems. In Nanomagnetism and Spintronics (ed. Shinjo T.). Elsevier, Amsterdam, pp. 155-187.

43) Ono, T., Miyajima, H., Shigeto, K., Mibu, K., Hosoito, N. and Shinjo, T. (1999) Propagation of a magnetic domain wall in a submicron magnetic wire. Science 284, 468-470.

44) Yamaguchi, A., Ono, T., Nasu, S., Miyake, K., Mibu, K. and Shinjo, T. (2004) Real-space observation of current-driven domain wall motion in submicron magnetic wires. Phys. Rev. Lett. 92, 077205077207.

45) Tanabe, S., Miwa, S., Mizuguchi, M., Shinjo, T., Suzuki, Y. and Shiraishi, M. (2007) Spin dependent transport in nanocomposites of Alq3 molecules and Co nanoparticles. Appl. Phys. Lett. 91,
63123-63126.

46) Miwa, S., Shiraishi, M., Tanabe, S., Mizuguchi, M., Shinjo, T. and Suzuki, Y. (2007) Tunneling magnetoresistance of $\mathrm{C}_{60}$-Co nanocomposites and spin dependent transport in organic semiconductors. Phys. Rev. B 76, 214414-214417.

47) Nozaki, T., Shiota, Y., Shiraishi, M., Shinjo, T. and Suzuki, Y. (2010) Voltage induced perpendicular magnetic anisotropy change in magnetic tunnel junctions. Appl. Phys. Lett. 96, 022504-022507.

48) Shiota, Y., Nozaki, T., Bonnel, F., Murakami, S., Shinjo, T. and Suzuki, Y. (2012) Induction of coherent magnetization switching in a few atomic layers of FeCo using voltage pulses. Nat. Mater. 11, 39-43.

(Received Sep. 28, 2012; accepted Nov. 1, 2012)

\section{Profile}

Teruya Shinjo was born in 1938. He graduated from Kyoto University in 1961 and received a $\mathrm{PhD}$ in 1966. He joined the Institute for Chemical Research, Kyoto University as a research associate in 1966, and became an associate professor in 1976, and a full professor in 1982. From 1974 to 1975 , he was a guest scientist at Universität des Saarlandes, West Germany. From 1992 to 2000, he served as a science advisor to the Japanese Ministry of Education, Science, Sports and Culture. From 1996 to 1998, he served as the director of the Institute for Chemical Research, Kyoto University. In 2002, he retired from Kyoto University and became a professor emeritus. Since 2002, he has been a specially-appointed professor at Osaka University and a senior researcher at the International Institute for Advanced Studies.

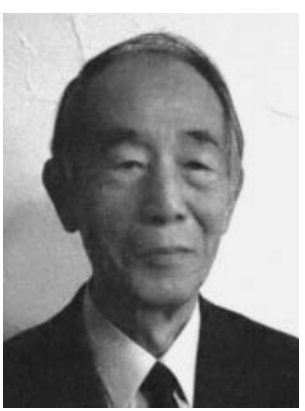

In 2006, he received a Doctor Honoris Causa title from the Technical University of Ostrava, the Czech Republic. He is currently serving as a vice president of the Foundation Advanced Technology Institute. He received the Award of the Society of Applied Physics (1993), the Award of the Japan Magnetics Society (1997), the Medal with Purple Ribbon (2001), the Kotaro Honda Memorial Award (2007) and the Toray Science and Technology Prize (2009). 